\title{
Dynamical Fano resonance of an exciton in laser-driven semiconductor superlattices
}

\author{
Nobuya Maeshima ${ }^{1,2, *}$ and Ken-ichi Hino ${ }^{2,1}$ \\ ${ }^{1}$ Center for Computational Sciences, University of Tsukuba, Tsukuba 305-8577, Japan \\ ${ }^{2}$ Division of Materials Science, Faculty of Pure and Applied Sciences, University of Tsukuba, Tsukuba 305-8573, Japan
}

(Received 13 January 2012; revised manuscript received 18 April 2012; published 7 May 2012)

\begin{abstract}
An excitonic Floquet state in semiconductor superlattices (SLs) driven by an intense monochromatic laser is examined based on multichannel scattering theory, where this state is supported by a photon sideband attributed to a SL joint miniband. It is shown that the excitonic Fano resonance (FR) is caused by the coupling between photon sidebands, and thus one obtains the possibility of dynamic quantum control of this exciton state by means of laser tuning. This FR differs from the conventional FR observed in the original SLs without laser irradiation in that the latter FR results from the static Coulomb coupling between different SL joint minibands. Both the $q$ parameter and the spectral width $\Gamma$ associated with the excitonic FR of concern vary notably with a change of laser strength. In particular, it is found that the FR is made stable, that is, $1 /|q|$ and $\Gamma$ become minimized, when the laser strength is appropriately tuned to the condition of what is called dynamic localization.
\end{abstract}

DOI: 10.1103/PhysRevB.85.205305

PACS number(s): 78.67.Pt, 71.35.Cc, 78.20.Bh

\section{INTRODUCTION}

Nonlinear effects of ac electric fields on materials have been of great importance in studies aimed at revealing unexplored quantum dynamics and realizing the related coherent control. ${ }^{1,2}$ Phenomena caused by a temporally periodic drive are of particular interest; they can be analyzed theoretically by means of the Floquet formalism. ${ }^{3}$ In particular, the coherent destruction of tunneling ${ }^{4,5}$ (CDT) in double wells is a typical phenomenon which can easily be explained in this way. The quantum driven tunneling dynamics in this system can be controlled by means of tuning the strength and frequency of an applied monochromatic cw laser.

A pheomenon closely related to CDT in condensed matter physics is the dynamic localization ${ }^{6,7}$ (DL) manifested in semiconductor superlattices (SLs) with a temporally periodic ac electric field $F(t)=F_{\text {ac }} \cos (\omega t)$ at time $t$ with strength and frequency $F_{\mathrm{ac}}$ and $\omega$, respectively. The relation of DL to CDT can be understood by a unified analytic treatment, ${ }^{8}$ and further it can be discussed from the viewpoint of group theory and the Landau-Zener problem. ${ }^{9}$ In the present system of ac-field-driven SLs, the nonlinear effect of the ac field is renormalized to an effective miniband width given by $\Delta_{\text {eff }}=\Delta_{\text {bare }}\left|J_{0}(x)\right|{ }^{7}$ where $\Delta_{\text {bare }}$ is the bare miniband width, and $J_{0}(x)$ is the zeroth-order Bessel function of the first kind with $x=e F_{\text {ac }} d / \hbar \omega$, where $e, d$, and $\hbar$ represent the elementary charge, the lattice constant of the SLs, and Planck's constant divided by $2 \pi$, respectively. When $x$ is tuned so as to satisfy the equation $J_{0}(x)=0$, the effective miniband collapses, resulting in DL in a similar manner to CDT.

The first observation of DL was made on electron-doped semiconductor SLs driven by a terahertz $(\mathrm{THz})$ wave. ${ }^{10} \mathrm{In}$ addition to this, DL has also been observed in diverse physical systems such as a cold atomic gas in a one-dimensional optical lattice, ${ }^{11}$ an atomic Bose-Einstein condensate, ${ }^{12}$ and light in curved waveguide arrays. ${ }^{13-15}$ Further, DL has been discussed in double-quantum-dot molecules,${ }^{16}$ one-dimensional lattices under the influence of ac electric and magnetic fields, ${ }^{17}$ Cooper pairs in Josephson qubits, ${ }^{18}$ and strongly correlated electron systems. ${ }^{19}$
The recent progress in THz light sources has encouraged experimental studies of coherent control of excitonic states in undoped SLs with a relatively weak THz field. ${ }^{20-28}$ Further, the development of higher-power $\mathrm{THz}$ light sources with a peak intensity of the order of hundreds of $\mathrm{kV} / \mathrm{cm}$ and above $\mathrm{e}^{29-31}$ enables one to investigate the unexplored research area of the Floquet exciton under consideration here; this exciton is composed of a photodressed electron-hole pair, each carrier of which is generated by THz-wave irradiation. Thus far, a number of theoretical studies have been devoted to revealing the anomalous behavior of such excitonic states regarding negative absorption (optical gain). ${ }^{32-35}$

In this paper, we focus on the possibility of realizing Fano resonance (FR) of the Floquet exciton in a laser-driven SL system; hereafter, the Floquet exciton is also termed a photodressed exciton. Particular attention is paid to understanding how the DL affects the photodressed-excitonic FR. It is expected that the FR of concern is caused by a coupling between photon sidebands mediated by ac Zener tunneling, where the photon sidebands are attributed to the joint SL minibands of a pair of a conduction band and a valence band. Hereafter, these bands are labeled $c$ and $v$, respectively, and the coupling mediated by the ac Zener tunneling is termed ac Zener coupling (ac ZC). ${ }^{36}$ To see this situation in more detail, the manifold formation in the present system is schematically shown in Fig. 1, where two photon sidebands labeled as $\bar{\mu}$ and $\bar{\mu}^{\prime}$ are depicted. The manifold label $\bar{\mu}^{(\prime)}$ represents a group of indices $\left(b_{c}^{(\prime)}, b_{v}^{(\prime)}, n_{p}^{(\prime)}\right)$, where $b_{c}^{(\prime)}$ and $b_{v}^{(\prime)}$ are SL miniband indices belonging to the $c$ and $v$ bands, respectively, and $n_{p}^{(\prime)}$ is the photon number relevant to absorption and emission. When the effect of ac $\mathrm{ZC}$ is small, these indices are still considered as good quantum numbers. It is shown in Fig. 1 that the discrete photodressed-excitonic state labeled as EX is supported by the photon sideband $\bar{\mu}$, and, further, this is also embedded in the continuum of the alternative photon sideband $\bar{\mu}^{\prime}$. Thus, it is likely that FR occurs due to a close coupling between the two photon sidebands $\bar{\mu}$ and $\bar{\mu}^{\prime}$, and, eventually, the EX state decays into the continuum state that belongs to $\bar{\mu}^{\prime}$.

This coupling is classified into two types, that is, the ac ZC and the Coulomb coupling, in terms of the difference of the 


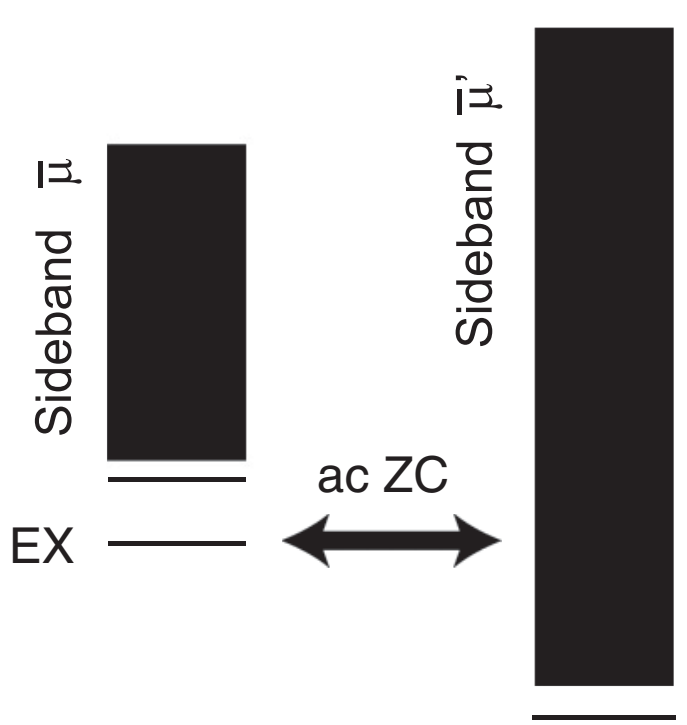

FIG. 1. Schematic diagram of manifold formation of the photodressed excitonic system. This shows the coupling mechanism in which the exciton state (EX) supported by the sideband $\bar{\mu}$ interacts with the continuum state belonging to the sideband $\bar{\mu}^{\prime}$ under ac ZC, leading to Fano decay.

photon numbers $\Delta n_{p}=\left|n_{p}-n_{p}^{\prime}\right|$. The former coupling has $\Delta n_{p} \neq 0$ and the latter coupling $\Delta n_{p}=0$. In the former type of FR, the spectral profile and intensity can be controlled to some extent by modulating the laser parameters $F_{\mathrm{ac}}$ and $\omega$ in an appropriate manner, since the strength of ac ZC depends exclusively on both the external parameters. On the other hand, in the latter type of FR, there is little possibility of such quantum control of the FR spectra, since it is considered difficult to modify the strength of the Coulomb interaction by means of the laser tuning. In this context, hereafter, the former type of FR is termed dynamic FR (DFR) in order to distinguish it from the latter type of static FR. When $F_{\mathrm{ac}}$ is weak enough to suppress the effect of ac ZC, the photodressed-excitonic FR is dominated by the Coulomb coupling. Its underlying physics can be understood as almost the same as that of the conventional FR observed in the original SLs without laser irradiation and in biased SLs with a weak bias field. ${ }^{37}$

As is shown later in detail, both the $q$ parameter (asymmetry parameter) and the spectral width $\Gamma$ characteristic of the excitonic DFR vary notably with the change of $F_{\text {ac }}$. In particular, it is found that $1 /|q|$ and $\Gamma$ are minimized when $F_{\mathrm{ac}}$ is appropriately tuned into the condition of DL, implying that the photodressed exciton is made stable with a long lifetime.

In the following, recent investigations related to the present study of DFR are briefly described and the differences among them clarified. For studies of a dynamic Wannier-Stark ladder, the terminology of DFR was used in different senses in Refs. 38 and 39. In the former study, the present authors and co-workers showed that a Floquet state of a single electron-differing from that of the exciton considered here-was unstable under a relatively intense $\mathrm{THz}$ field due to the ac $\mathrm{ZC}$, and, in particular, when DL occurred, its instability could be understood in terms of the Fano-type decay mechanism. This was termed DFR. It should be noted that such instability is inherent to the Wannier-Stark ladder, namely, biased SLs, with a relatively large bias field, as is discussed in Ref. 38. Thus, this DFR has no contribution in the present bias-free SL system. On the other hand, in the latter study, excitonic FR was dealt with. However, the applied $\mathrm{THz}$ wave was so weak that ac ZC was of no importance, and hence the FR was caused simply by a Coulomb interaction.

In addition to these two studies, the nonlinear Fano effect was discussed in semiconductor quantum wells and quantum dots in Refs. 40 and 25, respectively, where the FR arising from static couplings was probed in an optically nonlinear regime at high power. This is somewhat similar to laser-induced autoionization, ${ }^{41,42}$ rather than to the laser-induced continuum structure $^{43}$ akin to the DFR discussed here.

This paper is organized as follows. Section II describes the theoretical framework. Section III presents the results and discussion. Finally, Sec. IV presents the conclusions. Atomic units (a.u.) are used throughout unless otherwise stated.

\section{THEORETICAL FRAMEWORK}

This section consists of two subsections. In Sec. II A, we derive the nontrivial expression for the optical absorption spectra of the present Floquet system driven by $F(t)$, where the spectra are probed by an alternative weak laser. ${ }^{35,44,45}$ This expression is obtained by solving the semiconductor Bloch equations. The resulting microscopic polarization function is expressed as a linear combination of the photodressedexcitonic states satisfying the Wannier equation. In Sec. II B, we show that the Wannier equation, namely, the excitonic Floquet equation, is reduced to the coupled equations for the multichannel scattering problem relevant to the DFR of concern.

\section{A. Optical absorption spectra}

The total Hamiltonian of the system concerned comprises a SL Hamiltonian composed of field-free Hamiltonians for the $c$ and the $v$ bands, a Coulomb interaction between electrons, an intersubband interaction caused by the driving laser $F(t)$, and an interband interaction invoked by the probe laser $f_{p}(t)$, where $f_{p}(t)=f_{p 0} \cos \left(\omega_{p} t\right)$ with $f_{p 0}$ the amplitude and $\omega_{p}$ the frequency. The formulation developed here is directed toward seeking the nontrivial time evolution of the microscopic polarization of the interband transition (the off-diagonal part of the density matrix). This is obtained by solving the Liouville equation of the present system, based on a similar theoretical framework to that in Ref. 44. The microscopic polarization is defined as $p_{\lambda \lambda^{\prime} \boldsymbol{K}}(t) \equiv\left\langle a_{\lambda \boldsymbol{K}}^{(v) \dagger} a_{\lambda^{\prime} \boldsymbol{K}}^{(c)}\right\rangle$, where $\langle O\rangle$ represents the expectation value of the operator $O$. Here, $\lambda^{(\prime)}$ represents the joint SL miniband index $b^{(\prime)}$ and the SL site $l^{(\prime)}$, namely, $\lambda^{(\prime)}=\left(b^{(\prime)}, l^{(\prime)}\right)$, and $\boldsymbol{K}$ is the in-plane momentum associated with the relative motion of the pair of a $c$-band and a $v$-band electron; in plane is defined as in the plane normal to the direction of crystal growth (the $z$ axis). Further, $a_{\lambda \boldsymbol{K}}^{(s) \dagger}\left(a_{\lambda \boldsymbol{K}}^{(s)}\right)$ represents the creation (annihilation) operator of the electron with $\lambda$ and $\boldsymbol{K}$ in the band $s$.

The main approximations made here are summarized in the following; for more detail, consult Ref. 44, in which the validity and criteria of applicability of the approximations are also discussed. (i) The nearest-neighbor tight-binding model 
for the $c$ - and $v$-band SL Hamiltonians is employed. (ii) The Wannier function expressed as $\langle z \mid \lambda\rangle$ is approximated by the corresponding wave function of a single quantum well with an infinite potential barrier, where $\langle z \mid \lambda\rangle$ represents the function at the position $z-l d$ in miniband $b$ with $d$ the lattice constant of the concerned SLs. (iii) Just the Coulomb interaction for an exciton composed of only a single pair of a $c$-band and $v$-band electron is retained, and the many-body Coulomb correlation effect is neglected. (iv) It is assumed that the probe laser is weak enough to satisfy the relation $F_{\text {ac }} \gg f_{p 0}$ and that $\omega_{p}$ is much greater than $\omega$, namely, $\omega \ll \omega_{p}$. Hence, the resulting absorption coefficient is linear in $f_{p}(t)$ but nonlinear in $F(t)$.

We begin with the equation of motion for the microscopic polarization given by

$$
i \frac{\partial}{\partial t} p_{\lambda \lambda^{\prime} \boldsymbol{K}}=\left\langle\left[a_{\lambda \boldsymbol{K}}^{(v) \dagger} a_{\lambda \boldsymbol{K}}^{(c)}, H\right]\right\rangle,
$$

where $H$ is the total Hamiltonian under consideration. By making the approximation (iii) in addition to the other approximations mentioned above and using the rotating wave approximation for the probe laser, the above equation becomes the semiconductor Bloch equation for the polarization $\bar{p}\left(\rho, z_{v}, z_{c}, t\right)$ as follows:

$$
\begin{aligned}
\left(i \frac{\partial}{\partial t}+i \gamma+\omega_{p}\right) \bar{p}\left(\boldsymbol{\rho}, z_{v}, z_{c}, t\right) \\
+(2 \pi)^{2} e^{i \omega_{p} t} f_{p}^{(+)}(t) d_{0}^{(v c)} \delta(\rho) \delta\left(z_{v}-z_{c}\right) \\
=\int d z\left[\bar{p}\left(\rho, z_{v}, z, t\right) H_{\mathrm{TB}}^{(c)}\left(z, z_{c}, t\right)\right. \\
\left.\quad \int H_{\mathrm{TB}}^{(v)}\left(z_{v}, z, t\right) \bar{p}\left(\boldsymbol{\rho}, z, z_{c}, t\right)\right] \\
\quad+\mathcal{H}\left(\boldsymbol{\rho}, z_{v}, z_{c}\right) \bar{p}\left(\boldsymbol{\rho}, z_{v}, z_{c}, t\right),
\end{aligned}
$$

where $\bar{p}\left(\boldsymbol{\rho}, z_{v}, z_{c}, t\right)$ is defined in the real-space representation as

$$
\begin{aligned}
\bar{p}\left(\boldsymbol{\rho}, z_{v}, z_{c}, t\right)= & e^{i \omega_{p} t} \sum_{\lambda, \lambda^{\prime}} \int d \boldsymbol{K} e^{i \boldsymbol{K} \cdot \boldsymbol{\rho}}\left\langle z_{v} \mid \lambda\right\rangle \\
& \times p_{\lambda \lambda^{\prime} \boldsymbol{K}}(t)\left\langle\lambda^{\prime} \mid z_{c}\right\rangle .
\end{aligned}
$$

Here, $d_{0}^{(v c)}$ is the interband dipole moment of the bulk material, $f_{p}^{(+)}(t) \equiv\left(f_{p} / 2\right) e^{-i \omega_{p} t}$, and $\gamma$ is an infinitesimal positive number. The Hamiltonian given by

$$
\mathcal{H}\left(\boldsymbol{\rho}, z_{v}, z_{c}\right)=-\frac{\nabla_{\boldsymbol{\rho}}^{2}}{2 m_{\|}}+V\left(\boldsymbol{\rho}, z_{v}, z_{c}\right)
$$

is composed of a kinetic-energy term in the $\rho$ plane and the Coulomb interaction

$$
V\left(\rho, z_{v}, z_{c}\right)=-\frac{1}{\epsilon \sqrt{\rho^{2}+\left(z_{v}-z_{c}\right)}},
$$

with $m_{\|}$and $\epsilon$ as the in-plane reduced mass and static dielectric constant, respectively, and with $\rho=|\rho|$.

The tight-binding Hamiltonian in Eq. (2) is given by

$$
H_{\mathrm{TB}}^{(s)}\left(z, z^{\prime}, t\right) \equiv\left\langle z\left|\hat{H}_{\mathrm{TB}}^{(s)}(t)\right| z^{\prime}\right\rangle
$$

in the real-space representation, where

$$
\begin{aligned}
\hat{H}_{\mathrm{TB}}^{(s)}(t)= & \sum_{\lambda=(b, l)}\left[\epsilon_{0 b}^{(s)}|\lambda\rangle\langle\lambda|+\left\{(-1)^{b+\sigma^{s}} / 4\right\}\right. \\
& \left.\times \Delta_{b}^{(s)}(|l, b\rangle\langle l+1, b|+| l+1, b\rangle\langle l, b|)\right] \\
& -F(t) \sum_{\lambda \lambda^{\prime}}|\lambda\rangle Z_{\lambda \lambda^{\prime}}\left\langle\lambda^{\prime}\right|,
\end{aligned}
$$

with $\epsilon_{0 b}^{(s)}$ and $\Delta_{b}^{(s)}$ as the band center and the width of $b$, respectively, and, further, $\sigma^{s}=0$ (for $s=c$ ) and 1 (for $s=v$ ). The last term of Eq. (7) represents the dipole interaction induced by the driving laser with $Z_{\lambda, \lambda^{\prime}}$ as a dipole matrix element. It should be noted that the off-diagonal contribution of $Z_{\lambda, \lambda^{\prime}}$ with $b \neq b^{\prime}$ induces the ac ZC to be highlighted below.

The concerned function $\bar{p}\left(\rho, z_{v}, z_{c}, t\right)$ can be expressed by use of the complete set of Floquet wave functions $\psi_{E \beta}\left(\boldsymbol{\rho}, z_{v}, z_{c}, t\right)$ (for more details, see the Appendix), satisfying

$$
\begin{aligned}
\left(i \frac{\partial}{\partial t}+E\right) \psi_{E \beta}\left(\boldsymbol{\rho}, z_{v}, z_{c}, t\right) \\
=\int d z\left[\psi_{E \beta}\left(\boldsymbol{\rho}, z_{v}, z, t\right) H_{\mathrm{TB}}^{(c)}\left(z, z_{c}, t\right)\right. \\
\left.\quad-H_{\mathrm{TB}}^{(v)}\left(z_{v}, z, t\right) \psi_{E \beta}\left(\boldsymbol{\rho}, z, z_{c}, t\right)\right] \\
\quad+\mathcal{H}\left(\boldsymbol{\rho}, z_{v}, z_{c}\right) \psi_{E \beta}\left(\boldsymbol{\rho}, z_{v}, z_{c}, t\right),
\end{aligned}
$$

since this is read as the homogeneous equation corresponding to the inhomogeneous equation (2). Here, the temporally periodic boundary condition

$$
\psi_{E \beta}\left(\rho, z_{v}, z_{c}, t\right)=\psi_{E \beta}\left(\rho, z_{v}, z_{c}, t+T\right)
$$

is imposed on Eq. (8), and the orthogonality relation

$$
\begin{aligned}
& \frac{1}{T} \int_{0}^{T} d t \int d \rho \int d z_{v} \int d z_{c} \psi_{E \beta}^{*}\left(\boldsymbol{\rho}, z_{v}, z_{c}, t\right) \\
& \quad \times \psi_{E^{\prime} \beta^{\prime}}\left(\boldsymbol{\rho}, z_{v}, z_{c}, t\right)=\delta\left(E-E^{\prime}\right) \delta_{\beta \beta^{\prime}}
\end{aligned}
$$

is ensured, with $E^{(\prime)}$ and $T=2 \pi / \omega$ as a quasienergy and the time period of the driving laser field, respectively. Equation (8) is nothing but the Wannier equation of the photodressed exciton of concern. It should be noted that this is cast into the multichannel scattering equations, as shown in Sec. II B, and hence the Floquet wave function of $\psi_{E \beta}\left(\rho, z_{v}, z_{c}, t\right)$ forms a continuum spectrum designated by both $E$ and $\beta$ representing the label of an open channel. This multichannel feature is brought about by the strong driving laser $F(t)$ that closely couples an excitonic bound state with continua.

By using Eq. (10), $\bar{p}\left(\rho, z_{v}, z_{c}, t\right)$ can be expanded as

$$
\bar{p}\left(\boldsymbol{\rho}, z_{v}, z_{c}, t\right)=\int d E \sum_{\beta} a_{E \beta} \psi_{E \beta}\left(\boldsymbol{\rho}, z_{v}, z_{c}, t\right),
$$

and immediately the expansion coefficient $a_{E \beta}$ is obtained as

$$
a_{E \beta}=\frac{(2 \pi)^{2} d_{0}^{(v c)}}{\left(E-\omega_{p}-i \gamma\right) T} \int_{0}^{T} d t^{\prime} \bar{\psi}_{E \beta}\left(t^{\prime}\right),
$$

where

$$
\bar{\psi}_{E \beta}(t)=\int d z \psi_{E \beta}(\mathbf{0}, z, z, t)
$$


The linear optical susceptibility $\chi(t)$ with respect to $f_{p}(t)$ is provided by 44

$$
\chi(t)=\frac{\left|d_{0}^{(v c)}\right|^{2}}{\epsilon_{0}} \int d E \sum_{\beta} \frac{O_{E \beta}(t)}{E-\omega_{p}-i \gamma},
$$

where

$$
O_{E \beta}(t)=\bar{\psi}_{E \beta}(t) \frac{1}{T} \int_{0}^{T} d t^{\prime} \bar{\psi}_{E \beta}^{*}\left(t^{\prime}\right)
$$

and $\epsilon_{0}$ is the dielectric constant of vacuum. By expressing $\chi(t)$ as the Fourier-series expansion

$$
\chi(t) \equiv \sum_{j} e^{i j \omega t} \chi_{j}\left(\omega_{p} ; \omega\right),
$$

the absorption coefficient to be calculated is given in terms of the imaginary part of the Fourier coefficient $\chi_{j}\left(\omega_{p} ; \omega\right)$ by

$$
\alpha\left(\omega_{p} ; \omega\right)=\frac{\omega_{p}}{c} \sum_{j} \operatorname{Im} \chi_{j}\left(\omega_{p} ; \omega\right)
$$

with $c$ representing the speed of light. Taking account of the Fourier-series expansion

$$
O_{E \beta}(t)=\sum_{j} e^{i j \omega t} \tilde{O}_{E \beta j},
$$

$\alpha\left(\omega_{p} ; \omega\right)$ ends up as

$$
\alpha\left(\omega_{p} ; \omega\right)=\pi \frac{\omega_{p}\left|d_{0}^{(v c)}\right|^{2}}{c \epsilon_{0}} \sum_{\beta j} \tilde{O}_{\omega_{p} \beta j},
$$

where the limit of $\gamma \rightarrow+0$ is taken.

\section{B. Multichannel scattering equations of the photodressed exciton}

The next task is to solve Eq. (8) and obtain the set of wave functions, $\left\{\psi_{E \beta}\left(\rho, z_{v}, z_{c}, t\right)\right\}$, followed by the evaluation of Eq. (13) and then Eq. (17). With this aim, first, we expand $\psi_{E \beta}\left(\rho, z_{v}, z_{c}, t\right)$ as

$$
\psi_{E \beta}\left(\rho, z_{v}, z_{c}, t\right)=\sum_{\mu} \Phi_{\mu}\left(z_{v}, z_{c}, t\right) F_{\mu \beta}(\rho),
$$

where the argument of the function of $F_{\mu \beta}$ is considered as $\rho$ rather than $\rho$, since higher-angular-momentum components than the $s$ component to be taken here have no effect on Eq. (13). In Eq. (20), $\Phi_{\mu}\left(z_{v}, z_{c}, t\right)$ is the real-space representation of the Floquet state $\left|\Phi_{\mu}(t)\right\rangle$, that is, $\Phi_{\mu}\left(z_{v}, z_{c}, t\right)=$ $\left\langle z_{v}, z_{c} \mid \Phi_{\mu}(t)\right\rangle$, satisfying

$$
\left[\hat{H}_{\mathrm{TB}}-i \frac{\partial}{\partial t}\right]\left|\Phi_{\mu}\right\rangle=\mathcal{E}_{\mu}\left|\Phi_{\mu}\right\rangle,
$$

where

$$
\hat{H}_{\mathrm{TB}} \equiv \hat{H}_{\mathrm{TB}}^{(c)}+\hat{H}_{\mathrm{TB}}^{(v)},
$$

and $\mathcal{E}_{\mu}$ is the $\mu$ th quasienergy. It is noted that the index of $\mu$ is considered as an approximate quantum number consisting of the composite quantum number of $\bar{\mu}$ introduced in Sec. I and the Bloch momentum $k$ of the joint miniband of $\left(b_{c}, b_{v}\right)$, that is, $\mu \approx[\bar{\mu}, k] \equiv\left[b_{c}, b_{v}, n_{p}, k\right]$. As mentioned in Sec. I, $\bar{\mu}$ becomes a set of good quantum numbers as $F_{\text {ac }}$ decreases. On the other hand, the quantum number $k$ always remains conserved, since Eq. (21) ensures spatial periodicity. To be specific, we bear in mind the Houston representation that is provided by the appropriate gauge transformation of Eq. (21). ${ }^{44}$

In view of Eq. (20), the Wannier equation of Eq. (8) can be reduced to the coupled equations for the radial wave function $F_{\nu \beta}(\rho)$,

$$
\sum_{\mu} L_{\mu \nu} F_{\nu \beta}(\rho)=E F_{\mu \beta}(\rho),
$$

where $L_{\mu \nu}$ is the operator given by

$$
L_{\mu \nu}=\delta_{\mu \nu}\left[-\frac{1}{2 m_{\|}}\left(\frac{d^{2}}{d \rho^{2}}+\frac{1}{\rho} \frac{d}{d \rho}\right)+\mathcal{E}_{\mu}\right]+V_{\mu \nu}(\rho) .
$$

The matrix element of the Coulomb interaction $V_{\mu \nu}(\rho)$ is defined as

$$
\begin{aligned}
V_{\mu \nu}(\rho)= & \frac{1}{T} \int_{0}^{T} d t \int d z_{v} \int d z_{c} \Phi_{\mu}^{*}\left(z_{v}, z_{c}, t\right) V\left(\rho, z_{v}, z_{c}\right) \\
& \times \Phi_{v}\left(z_{v}, z_{c}, t\right) .
\end{aligned}
$$

Equations (23) are considered as the multichannel scattering equations, because $V_{\mu \nu}(\rho) \sim 0$ at $\rho \gg 1$. Actually, for a given $E$, the channel $\mu$ satisfying $E>\mathcal{E}_{\mu}$ is an open channel, while the channel $\mu$ satisfying $E<\mathcal{E}_{\mu}$ is a closed channel. Thus, the label $\mu$ of $F_{\mu \beta}$ plays the role of a scattering channel. On the other hand, there are the same number of independent solutions as open channels, since as many scattering boundary conditions are imposed on the present problem in the asymptotic region of $\rho$. Here, the label $\beta$ of $F_{\mu \beta}$ means the number of independent solutions satisfying Eq. (23), as mentioned in Sec. II A.

Equation (23) can be numerically evaluated by virtue of the $R$-matrix propagation method. It is demonstrated that this sophisticated formalism provides a stable numerical algorithm with extremely high accuracy. Lately, this method has been applied to semiconductor nanostructures to investigate their optical properties, ${ }^{38,46,47}$ in addition to the conventional applications to the studies of electronic collisions with atomic and molecular targets and chemical reactions. Aside from the above-mentioned advantages, in general, the algorithm requires large-scale computation to obtain physical quantities such as spectral functions. In order to remedy this difficulty, intensive efforts have been made to develop a parallelization of the $R$-matrix formalism. ${ }^{48-50}$ Recently, the present authors have explored a parallelization scheme from a different point of view than the conventional schemes. ${ }^{45}$ The algorithm thus developed is fully applied to the present photodressedexcitonic problem. For more detail of the framework of this parallelization and the resulting degree of numerical efficiency, consult Ref. 45.

\section{RESULTS AND DISCUSSION}

This section consists of two subsections. First of all, in Sec. III A, we present the calculated absorption spectra, followed by discussing the overall spectral alteration with respect to $F_{\text {ac }}$. In $\mathrm{Sec}$. III B, we pay particular attention to the spectra with a Fano-type line shape manifested in the relatively large $F_{\text {ac }}$ region; this is DFR of the photodressed 


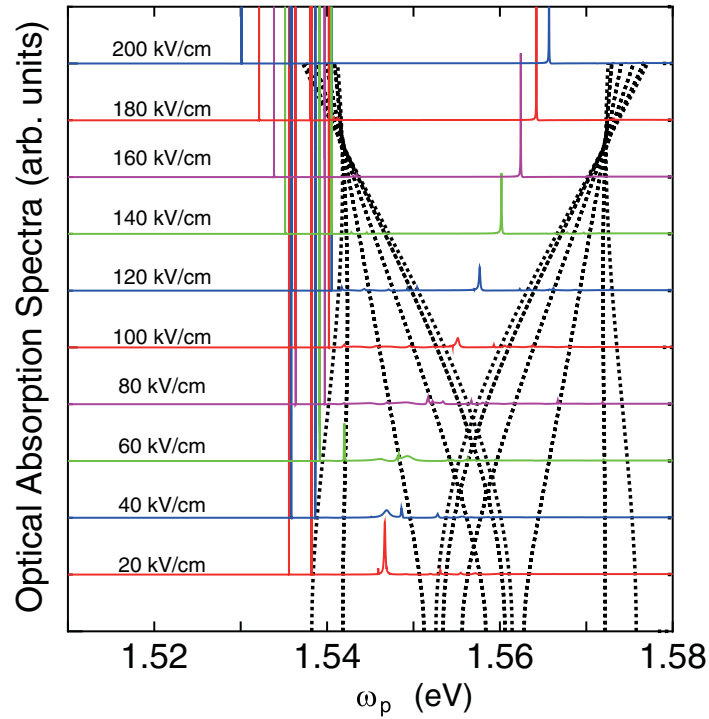

FIG. 2. (Color online) The optical absorption spectra $\alpha\left(\omega_{p} ; \omega\right)$ as a function of $\omega_{p}$ for $F_{\mathrm{ac}}=10-200 \mathrm{kV} / \mathrm{cm}$ (solid lines), where the colored traces are shifted vertically to enhance the presentation. Further, the quasienergies $\mathcal{E}_{\mu}$ as a function of $F_{\text {ac }}$ are also included (dotted lines). For more detail, consult the text.

exciton arising from the mechanism shown in Fig. 1. We also discuss the effect of DL on the DFR.

\section{A. Overall spectral alteration with respect to $F_{\text {ac }}$}

Numerical calculations are done for semiconductor SLs composed of ten quantum wells by employing the same physical parameters as those in Refs. 35 and 47, where each quantum well consists of a 9.9-nm-thick well layer of GaAs and a 3.1-nm-thick barrier layer of $\mathrm{Ga}_{0.75} \mathrm{Al}_{0.25} \mathrm{As}$. In the present calculations, the joint SL minibands of $\left(b_{c}, b_{v}\right)=(1,1)$ and $(2,1)$, and the associated manifolds of $\left(b_{c}, b_{v}, n_{p}\right)=$ $\left(1,1, n_{p}\right)$ and $\left(2,1, n_{p}\right)$ with $n_{p}=-3-3$ are included. Here, the frequency $\omega$ is set equal to $91 \mathrm{meV}$, which is identical to the difference between the centers of the joint minibands $(1,1)$ and $(2,1)$.

Figure 2 shows the calculated natural spectra of $\alpha\left(\omega_{p} ; \omega\right)$ as a function of $\omega_{p}$ in the range of $F_{\mathrm{ac}}=20-200 \mathrm{kV} / \mathrm{cm}$. The structure of the quasienergy $\mathcal{E}_{\mu}$ of the two photon sidebands with $\bar{\mu}=(1,1,0)$ and $(2,1,-1)$ is superimposed on these spectra, where the values of $\mathcal{E}_{\mu}$ are given on the abscissa as a function of $F_{\text {ac }}$. Since one-photon resonance between the joint miniband centers of $(1,1)$ and $(2,1)$ takes place, the bandwidth of $\mathcal{E}_{[1,1,0, k]}$ overlaps that of $\mathcal{E}_{[2,1,-1, k]}$ at $F_{\text {ac }}=0$, as seen in Fig. 2, where the former narrower sideband is situated around $\omega_{p}=1.552-1.563 \mathrm{eV}$, while the latter wider sideband is around $\omega_{p}=1.538-1.576 \mathrm{eV}$. Hereafter, the sidebands $(1,1,0)$ and $(2,1,-1)$ are termed $A$ and $B$, respectively. As $F_{\text {ac }}$ becomes more than approximately $70 \mathrm{kV} / \mathrm{cm}$, these two sidebands cause each other to curve, leading to splitting into two parts due to ac ZC. Thus, it is no longer possible to assign these sidebands simply in terms of the labels $A$ and $B$. Hereafter, the sideband on the higher-energy side and that on the lower-energy side are termed the upper and lower

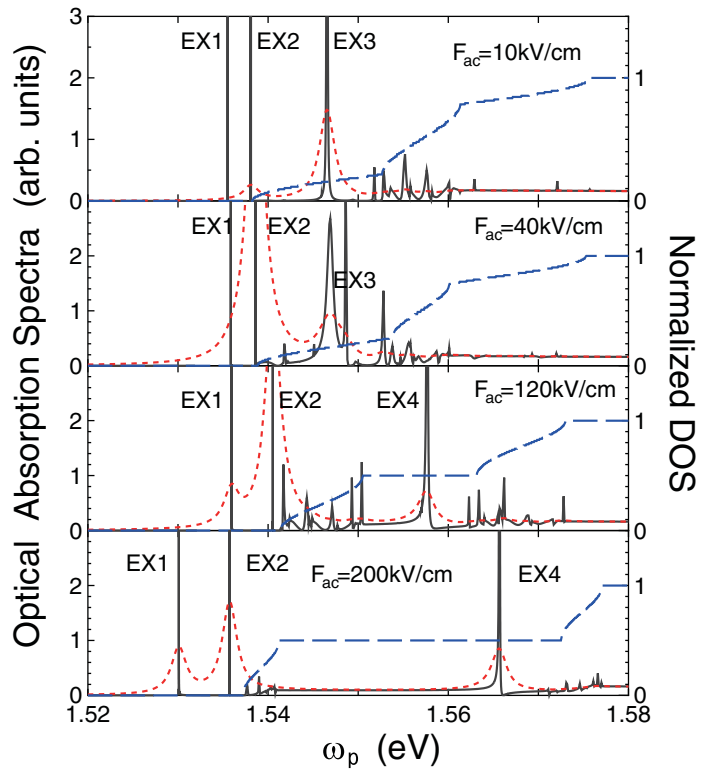

FIG. 3. (Color online) The optical absorption spectra as a function of $\omega_{p}$ at $F_{\mathrm{ac}}=10,40,120$, and $200 \mathrm{kV} / \mathrm{cm}$; the natural spectra $\alpha\left(\omega_{p} ; \omega\right)$ (solid lines) and their Lorentzian-convoluted forms $\tilde{\alpha}\left(\omega_{p} ; \omega\right)$ with homogeneous broadening of $1 \mathrm{meV}$ (red dotted lines). The normalized densities of states $\tilde{D}\left(\omega_{p}\right)$ for photodressed SLs are depicted by the blue dashed lines. The labels of EX $n$ with $n=1-4$ represent the spectral peaks relevant to characteristic photodressed excitonic states. For more detail, consult the text.

sidebands, respectively. It is noted that these two sidebands form DL around $F_{\mathrm{ac}} \approx 170 \mathrm{kV} / \mathrm{cm}$.

As is seen in the spectra at $F_{\mathrm{ac}}=20$ and $40 \mathrm{kV} / \mathrm{cm}$ of Fig. 2, sharp spectral peaks manifest themselves right below the lower edges of the sidebands of $A$ and $B$. On the analogy of excitonic spectra of field-free SLs,$^{37}$ it seems certain that these discernible peaks can be attributed to photodressed-excitonic states pertaining to nearby photon sidebands. In the greater$F_{\text {ac }}$ region, however, it is more difficult to determine whether the observed spectral peaks can be assigned to photodressedexcitonic states pertaining to the upper or to the lower sideband.

The four representative spectra at $F_{\mathrm{ac}}=10,40,120$, and $200 \mathrm{kV} / \mathrm{cm}$ are shown again in Fig. 3 to examine the spectral variation. The normalized density of states (DOS) defined as

$$
\tilde{D}\left(\omega_{p}\right)=\frac{\Delta D\left(\omega_{p}\right)}{\Delta D(1.58 \mathrm{eV})}
$$

is also depicted, where $\Delta D\left(\omega_{p}\right) \equiv D\left(\omega_{p}\right)-D(1.52 \mathrm{eV})$. Here, $D\left(\omega_{p}\right)$ represents the three-dimensional DOS of a free-electron pair belonging to the $c$ and $v$ bands, given by

$$
D\left(\omega_{p}\right)=D_{2 \mathrm{D}} \sum_{\mu} \Theta\left(\omega_{p}-\mathcal{E}_{\mu}\right),
$$

where $\Theta\left(\omega_{p}-\mathcal{E}_{\mu}\right)$ is the Heaviside function, and $D_{2 \mathrm{D}}$ is a constant representing the two-dimensional DOS of this pair associated with the relative motion in the $\rho$ plane. Obviously, $\tilde{D}\left(\omega_{p}\right)$ reflects the structure of the quasienergy $\mathcal{E}_{\mu}$, showing the ascending steplike shape. It is remarked that $\Delta D(1.58 \mathrm{eV})$ is constant and independent of $F_{\text {ac }}$ as long as $F_{\text {ac }} \leqslant 200 \mathrm{kV} / \mathrm{cm}$, as shown in Fig. 3, since all channels below $\omega_{p}=1.58 \mathrm{eV}$ 
are open, and hence the number of open channels remains unaltered; that is, $\tilde{D}(1.58 \mathrm{eV})=1$. Here, the sidebands $A$ and $B$ give equal contributions to this value of unity, and so do the upper and lower sidebands. For a given $\omega_{p}$, in general, $\tilde{D}\left(\omega_{p}\right)$ becomes larger with an increase in the number of open channels. For instance, in the lowest two panels of Fig. 3, $0<$ $\tilde{D}\left(\omega_{p}\right)<0.5$ if the channels relevant to the lower sideband are partially open, while $0.5<\tilde{D}\left(\omega_{p}\right)<1$ if the channels relevant to the upper sideband are partially open.

At $F_{\mathrm{ac}}=10$ and $40 \mathrm{kV} / \mathrm{cm}$, there are three sharp exciton peaks discerned at $\omega_{p}=1.536,1.538$, and $1.546 \mathrm{eV}$, which are labeled as EX1, EX2, and EX3, respectively. According to the discussion above, it is speculated that EX1 can be assigned to a $1 s$ state supported by the $B$ sideband, while EX 2 and EX 3 can be assigned to $1 s$ and $2 s$ states supported by the $A$ sideband; for more detail, consult the discussion below in this section. As $F_{\text {ac }}$ increases from 10 to $40 \mathrm{kV} / \mathrm{cm}$, the EX3 line profile becomes broadened due to the interaction with the continuum relevant to the $B$ sideband; the manner of this is similar to the DFR mechanism mentioned in Sec. I. With a still larger $F_{\text {ac }}$, the EX3 peak becomes faint, as shown in the spectra at $F_{\text {ac }}=60$ and $80 \mathrm{kV} / \mathrm{cm}$ of Fig. 2. At $F_{\text {ac }}=120$ and $200 \mathrm{kV} / \mathrm{cm}$, where the hybridized band composed of the $A$ and $B$ sidebands is split into the upper and lower sidebands, a new excitonic peak labeled EX4 appears between these two sidebands. It should be noted that the EX4 spectra show an asymmetric Fano profile with a peak followed by a dip; these are formed by the DFR mechanism shown in Fig. 1, and this excitonic state would be supported by the upper sideband, as is shown later in deeper discussion. Hereafter, it is understood that the location of the $\mathrm{EX} n$ peak is represented by $\mathcal{E}_{\mathrm{EX} n}$ with $n=1-4$.

Next, we discuss the difference between the DFR spectra of EX3 in the top two panels for $F_{\mathrm{ac}}=10$ and $40 \mathrm{kV} / \mathrm{cm}$, and those of EX4 in the bottom two panels for $F_{\mathrm{ac}}=120$ and $200 \mathrm{kV} / \mathrm{cm}$, where the former spectra look almost symmetric and the latter spectra look asymmetric. The shape of the DFR spectra is governed by the so-called $q$ parameter corresponding to the ratio of the contribution of a discrete level to that of the continuum background; with a decrease in $|q|$, the profile becomes more asymmetric, except in the very-small- $|q|$ region where a symmetric transparent window appears. As is seen in the spectra of EX3, the associated normalized DOS $\tilde{D}\left(\mathcal{E}_{\mathrm{EX} 3}\right)$ is still smaller than 0.5 , that is, the contribution of the continuum background is relatively small. Because of this fact, it is understood that the spectra are almost symmetric. On the other hand, as regards the DFR of EX4, the contribution of the continuum background is considered comparable to that of the excitonic discrete state, since all of the channels associated with the lower sideband are open; $\tilde{D}\left(\mathcal{E}_{\mathrm{EX} 4}\right)=0.5$. This is the reason why the DFR spectra of EX4 are asymmetric. More detailed discussion of the DFR of EX4 will be deferred to Sec. III B.

In Fig. 3, we also show the spectra provided by

$$
\tilde{\alpha}\left(\omega_{p} ; \omega\right)=\int d \omega_{p}^{\prime} f_{L}\left(\omega_{p}, \omega_{p}^{\prime}\right) \alpha\left(\omega_{p}^{\prime} ; \omega\right),
$$

where $\alpha\left(\omega_{p}^{\prime} ; \omega\right)$ is convoluted with a Lorentzian function defined by $f_{L}\left(\omega_{p}, \omega_{p}^{\prime}\right) \equiv(\delta / \pi) /\left[\left(\omega_{p}-\omega_{p}^{\prime}\right)^{2}+\delta^{2}\right]$. Here, the value of $\delta$ is set equal to $1 \mathrm{meV}$, corresponding to a realistic homogeneous broadening. For $F_{\mathrm{ac}}=10 \mathrm{kV} / \mathrm{cm}, \tilde{\alpha}\left(\omega_{p} ; \omega\right)$

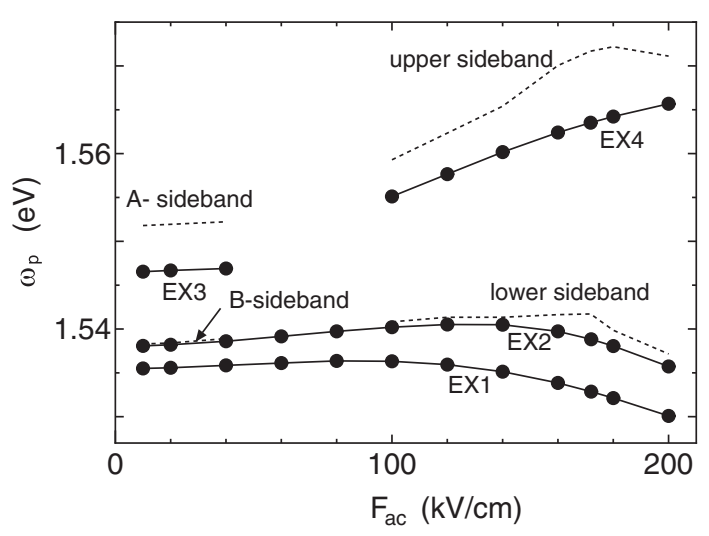

FIG. 4. The peak positions of the photodressed excitonic states EX $n$ with $n=1-4$ as a function of $F_{\text {ac }}$. The labels have the same meaning as those in Fig. 3. The calculated results represented by the filled circles are connected by the solid lines in order to aid the presentation. The lower bounds (absorption edges) of the photon sidebands of $A$ and $B$ and those of the upper and lower sidebands are also depicted by the dotted lines.

shows only two peaks pertaining to EX2 and EX3, whereas the spectrum of EX1 is no longer discernible, presumably due to the relatively weak ac $\mathrm{ZC}$ between the two sidebands $A$ and $B$. As is seen in this panel of Fig. 3, the peak height of $\tilde{\alpha}\left(\mathcal{E}_{\mathrm{EX} 2} ; \omega\right)$ is much smaller than that of $\tilde{\alpha}\left(\mathcal{E}_{\mathrm{EX} 3} ; \omega\right)$. In fact, this tiny contribution of EX2 can be attributed just to numerical inaccuracy, since it is speculated that the energy mesh, namely, $5 \times 10^{-2} \mathrm{meV}$, adopted in our numerical calculation is still larger than the natural width of the EX2 spectrum. That is, the excitonic state of EX2 is considered as a pure bound state rather than a DFR state, differing from that of EX3. This statement is confirmed by the fact that $\tilde{D}\left(\mathcal{E}_{\mathrm{EX} 3}\right) \approx 0$. For $F_{\mathrm{ac}}=40 \mathrm{kV} / \mathrm{cm}$, the natural width of EX2 grows, so that the spectra can be more accurately evaluated in our calculation. Actually, $\tilde{\alpha}\left(\mathcal{E}_{\mathrm{EX} 2} ; \omega\right)$ turns out to be larger than $\tilde{\alpha}\left(\mathcal{E}_{\mathrm{EX} 3} ; \omega\right)$, as is expected. This is the key reason why the EX2 peak is assigned to the $1 s$ state belonging to the manifold of $A$ rather than the $2 s$ state belonging to the manifold of $B$.

As $F_{\mathrm{ac}}$ further increases, $\tilde{\alpha}\left(\mathcal{E}_{\mathrm{EX} 1} ; \omega\right)$ becomes gradually larger, and at $F_{\mathrm{ac}}=120 \mathrm{kV} / \mathrm{cm}$, a small shoulder appears due to the mixing between the upper and lower sidebands, while $\tilde{\alpha}\left(\mathcal{E}_{\mathrm{EX} 3} ; \omega\right)$ almost disappears. As regards the DFR spectra of EX4 at $F_{\text {ac }}=120$ and $200 \mathrm{kV} / \mathrm{cm}$, the asymmetric profile found in $\alpha\left(\mathcal{E}_{\mathrm{EX} 4} ; \omega\right)$ is blurred to some extent in the more realistic spectrum of $\tilde{\alpha}\left(\mathcal{E}_{\mathrm{EX} 4} ; \omega\right)$. Therefore, it would be difficult to observe such asymmetry in actual experiments, as it stands. This issue will be mentioned again in Sec. IV.

Figure 4 shows $\mathcal{E}_{\mathrm{EX} n}(n=1-4)$ as a function of $F_{\mathrm{ac}}$. With an increase in $F_{\mathrm{ac}}$, a redshift of the peak positions of $\mathcal{E}_{\mathrm{EX} 1}$ and $\mathcal{E}_{\mathrm{EX} 2}$ is obvious, aside from the transient blueshift in the intermediate region of $F_{\text {ac }}$. This is in agreement with the results of Ref. 35, in which variational calculations were implemented without taking account of the multichannel scattering nature of the present problem, and $\omega$ was set equal to $30 \mathrm{meV}$ so as to satisfy the condition of three-photon resonance between the joint-miniband centers of $A$ and $B$. In contrast, the peak position of $\mathcal{E}_{\mathrm{EX} 4}$ is blueshifted as $F_{\mathrm{ac}}$ increases in the region of $F_{\text {ac }} \geqslant 100 \mathrm{kV} / \mathrm{cm}$. 
It is noted that the evaluation of a binding energy is not always a manageable task in such a multichannel scattering problem, since if there are two or more closed channels, we cannot uniquely determine the threshold energy as the reference energy from which the binding energies are reckoned. In Fig. 4, the lower bounds of the $A$ and $B$ sidebands and those of the upper and lower sidebands are also indicated. In the region of $F_{\text {ac }} \leqslant 40 \mathrm{kV} / \mathrm{cm}$, where ac ZC is not dominant, the binding energies of the excitonic states of EX1, EX2, and EX3 will be provided by the difference between the lower edge of sideband $B$ and $\mathcal{E}_{\mathrm{EX} 1}$, the difference between the lower edge of sideband $A$ and $\mathcal{E}_{\mathrm{EX} 2}$, and the difference between the lower edge of the sideband $A$ and $\mathcal{E}_{\mathrm{EX} 3}$, respectively. On the other hand, in the region of $F_{\mathrm{ac}} \geqslant 100 \mathrm{kV} / \mathrm{cm}$, where ac ZC is dominant, it seems difficult to relate the excitonic states of $\mathcal{E}_{\mathrm{EX} 1}$ and $\mathcal{E}_{\mathrm{EX} 2}$ with either the upper sideband edge or the lower sideband edge on a one-to-one basis. If the excitonic state of $\mathcal{E}_{\mathrm{EX} 2}$ at $F_{\text {ac }}=200 \mathrm{kV} / \mathrm{cm}$ was supported by the upper sideband, the resulting binding energy would amount to $35 \mathrm{meV}$; this seems too large since the binding energy of a conventional undressed exciton is of the order of $10 \mathrm{meV}$. On the contrary, if this excitonic state was supported by the lower sideband, the resulting binding energy would be nothing but approximately $1 \mathrm{meV}$; this is of the same order as $\delta$ adopted in the calculation of $\tilde{\alpha}\left(\mathcal{E}_{\mathrm{EX} 2} ; \omega\right)$, which leads to the paradoxical conclusion that the EX2 peak would likely be blurred out against the fact that it is clearly discernible in Fig. 3. Further, in the intermediate region of $F_{\text {ac }}$ between 40 and $100 \mathrm{kV} / \mathrm{cm}$, it is impossible to unequivocally determine the sideband supporting the excitonic states of EX1 and EX2.

As regards the binding energy of the excitonic state of EX4, the situation differs greatly from the situation concerning EX1 and EX2 in the region of $F_{\text {ac }} \geqslant 100 \mathrm{kV} / \mathrm{cm}$, since the upper sideband works effectively as only one closed channel located above $\mathcal{E}_{\mathrm{EX} 4}$ within our calculations; indeed, higher closed channels are located far above this, at least by approximately the value of $\omega$. Therefore, it is concluded that the binding energy of the excitonic state of EX4, represented as $\Delta \mathcal{E}_{\mathrm{EX} 4}$ here, can be evaluated as the difference between the upper sideband edge and $\mathcal{E}_{\mathrm{EX} 4}$. As is seen in Fig. $4, \Delta \mathcal{E}_{\mathrm{EX} 4}$ becomes maximized in the vicinity of $F_{\text {ac }}=170 \mathrm{kV} / \mathrm{cm}$ where DL occurs; $\Delta \mathcal{E}_{\mathrm{EX} 4}$ amounts to approximately $10 \mathrm{meV}$. This fact is incompatible with the result of Ref. 51 for the binding-energy calculations in the dynamic Wannier-Stark ladder, although the ac $\mathrm{ZC}$ is neglected there.

\section{B. Dynamic Fano resonance}

A detailed discussion of DFR of EX4 is given here, following the discussion of its binding energy in the preceding section. Figure 5 shows $\alpha\left(\omega_{p} ; \omega\right)$ around $\omega_{p}=\mathcal{E}_{\mathrm{EX} 4}$ as a function of $\omega_{p}-\mathcal{E}_{\mathrm{EX} 4}$ for $F_{\mathrm{ac}}=140-200 \mathrm{kV} / \mathrm{cm}$. It is seen that all of these spectra show an asymmetric shape with a peak followed by a dip. Moreover, $\alpha\left(\omega_{p} ; \omega\right)$ vanishes when $\omega_{p}$ matches the energy of the dip, that is, a completely transparent window opens. This implies that the DFR of concern is predominantly formed by the interaction between one open channel (the lower sideband) and one closed channel (the upper sideband). ${ }^{52}$ Therefore, the calculated spectra can be fitted to the following Fano formula suitable for such a channel

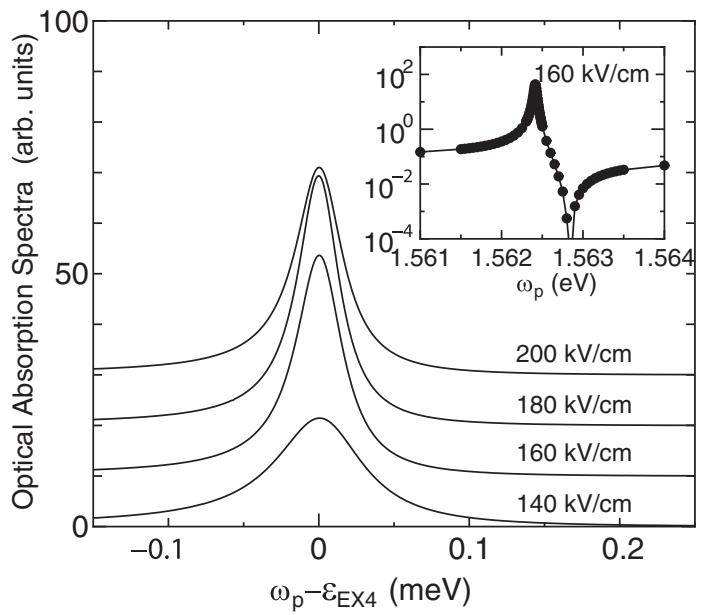

FIG. 5. The optical absorption spectra of $\alpha\left(\omega_{p} ; \omega\right)$ in the vicinity of $\mathcal{E}_{\mathrm{EX} 4}$ as a function of $\omega_{p}-\mathcal{E}_{\mathrm{EX} 4}$ for $F_{\mathrm{ac}}=140-200 \mathrm{kV} / \mathrm{cm}$, where the traces are shifted vertically to enhance the presentation. The inset shows the calculated results of $\alpha\left(\omega_{p} ; \omega\right)$ as a function of $\omega_{p}$ at $F_{\mathrm{ac}}=$ $160 \mathrm{kV} / \mathrm{cm}$ (filled circles) and the fitting curve in terms of Eq. (29) (solid line).

condition:

$$
\alpha\left(\omega_{p} ; \omega\right)=A \frac{\left(q+\bar{\omega}_{p}\right)^{2}}{1+\bar{\omega}_{p}^{2}},
$$

where $\bar{\omega}_{p}=\left(\omega_{p}-\mathcal{E}_{\mathrm{EX} 4}\right) /(\Gamma / 2)$ represents a normalized frequency, $q$ is the asymmetry parameter explained already in Sec. III A, and $A$ is a proportionality constant representing the transition probability of direct ionization of the photodressed exciton. In the inset of Fig. 5, it is demonstrated that the calculated results are perfectly in harmony with Eq. (29), where $q$ is negative.

According to this fitting procedure, the inverse of $q$, namely, $1 / q$, and the DFR width $\Gamma$ are obtained and are shown in Fig. 6 as a function of $F_{\text {ac }}$. These results clearly indicate that both of $1 / q$ and $\Gamma$ strongly depend on $F_{\text {ac }}$. This implies that

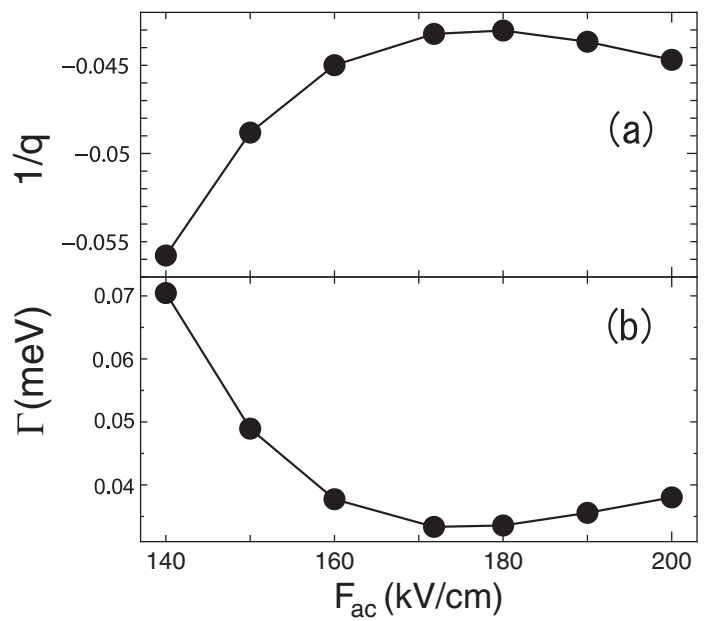

FIG. 6. (a) Inverse of the Fano line-shape parameter $q$ and (b) the natural spectral width of DFR, $\Gamma$, as functions of $F_{\mathrm{ac}}$. The calculated results represented by the filled circles are connected by the solid lines in order to aid the presentation. 
the modulation of laser intensity enables one to control the spectral profile as well as the binding energy of the excitonic DFR state. In particular, it should be noted that the minima of $|1 / q|$ and $\Gamma$ are located in the vicinity of $F_{\mathrm{ac}}=170 \mathrm{kV} / \mathrm{cm}$ where the DL condition is ensured. Actually, the DFR state with smaller $|1 / q|$ and $\Gamma$ is more similar to a pure bound state; both parameters vanish in the pure bound state, aside from a radiative decay that we are not concerned with here. Also, it should be brought to mind that the largest values of both $\Delta \mathcal{E}_{\mathrm{EX} 4}$ and $\alpha\left(\mathcal{E}_{\mathrm{EX} 4}, \omega\right)$ are realized around the DL, as shown in Figs. 4 and 5. In view of these facts, the DL is considered to fulfill the special role of quantum control of photodressed excitonic states.

\section{CONCLUSION}

The high-resolution optical absorption spectra of laserdriven SLs are calculated based on multichannel scattering theory by virtue of the $R$-matrix propagation method. The obtained photodressed-excitonic state shows the characteristic DFR structure induced by ac ZC. Further, the corresponding $q$ value, spectral width $\Gamma$, and binding energy $\Delta \mathcal{E}_{\mathrm{EX} 4}$ are altered with a change of $F_{\mathrm{ac}}$ in a relatively large $F_{\mathrm{ac}}$ region. In particular, in the vicinity of the DL condition, the photodressed-excitonic state becomes similar to a bound state rather than a resonance state. In other words, this state can be made more stable around the DL. These results add a further possibility of quantum control of excitons by means of laser irradiation, namely, dynamic quantum control.

Although we have succeed in bringing the DFR of the photodressed exciton to light, the spectral width of order less than $\delta$ still seems too small to be confirmed by experiments. In our calculations, the Coulomb many-body effect is neglected, as is mentioned in Sec. I. At the Hartree-Fock level, at least the self-energy correction to the electronic energy and the vertex correction to the Rabi energy have to be incorporated into the theory. ${ }^{53}$ It is straightforward to speculate that with an increase in $F_{\mathrm{ac}}$, both corrections will affect the DFR of concern more dominantly. To be specific, the larger effective Rabi energy attributed to the vertex correction would make the net ac ZC stronger, resulting in such a great DFR width that experimental measurement would be accessible. As regards the effect of the self-energy correction on DFR, the resonance position might be somewhat blueshifted, similarly to reported results in different systems. ${ }^{40}$ On the other hand, beyond the Hartree-Fock approximation, the spectral profile of DFR would be blurred to some extent by the dephasing due to photodressed exciton-exciton interactions. A study in this direction of the many-body effect is now in progress.

\section{ACKNOWLEDGMENTS}

This work was supported by a Grant-in-Aid for Scientific Research on Innovative Areas "Optical science of dynamically correlated electrons (DYCE)" (Grant No. 21104504) of the Ministry of Education, Culture, Sports, Science and Technology (MEXT), Japan. Numerical calculations were carried out on a T2K-Tsukuba System in the Center for Computational Sciences at the University of Tsukuba. The authors also thank K. Yashima and D. Misaki for their help in programming some of the algorithms into the computers.

\section{APPENDIX: FLOQUET FORMALISM}

In this appendix, we briefly introduce the Floquet formalism and the associated Floquet wave function which are used in this paper. Let us consider the wave function of a system driven by a temporally periodioc field with period $T$. Then the Schrödinger equation of the system is given by

$$
i \frac{\partial}{\partial t} \Psi(t)=\mathcal{H}(t) \Psi(t),
$$

where $\Psi(t)$ is the wave function of the system and the Hamiltonian $\mathcal{H}(t)$ has the time periodicity

$$
\mathcal{H}(t+T)=\mathcal{H}(t)
$$

Here spatial cordinates such as $z_{c}$ are omitted for simplicity. According to the Floquet theorem, there is a solution of Eq. (A1) in the form

$$
\Psi(t)=e^{-i E t} \psi(t),
$$

where $\psi(t)$ is the Floquet wave function, which satisfies the periodicity

$$
\psi(t+T)=\psi(t)
$$

and $E$ is the quasienergy. Substituting Eq. (A3) into Eq. (A1), we obtain an eigenvalue equation

$$
\left[\mathcal{H}(t)-i \frac{\partial}{\partial t}\right] \psi(t)=E \psi(t) .
$$

\footnotetext{
*maeshima@ims.tsukuba.ac.jp

${ }^{1}$ B. E. Cole, J. W. Williams, B. T. King, M. S. Sherwin, and C. R. Stanley, Nature (London) 410, 60 (2001).

${ }^{2}$ S. Kohler, J. Lehmann, and P. Hänggi, Phys. Rep. 406, 379 (2005), and references cited therein.

${ }^{3}$ J. H. Shirley, Phys. Rev. 138, B979 (1965).

${ }^{4}$ F. Grossmann, T. Dittrich, P. Jung, and P. Hänggi, Phys. Rev. Lett. 67, 516 (1991).

${ }^{5}$ M. Grifoni and P. Hänggi, Phys. Rep. 304, 229 (1998).

${ }^{6}$ D. H. Dunlap and V. M. Kenkre, Phys. Rev. B 34, 3625 (1986).
}

\footnotetext{
${ }^{7}$ M. Holthaus, Phys. Rev. Lett. 69, 351 (1992).

${ }^{8}$ M. Holthaus, Z. Rev. B 89, 251 (1992).

${ }^{9}$ Y. Kayanuma and K. Saito, Phys. Rev. A 77, 010101(R) (2008).

${ }^{10}$ B. J. Keay, S. Zeuner, S. J. Allen Jr., K. D. Maranowski, A. C. Gossard, U. Bhattacharya, and M. J. W. Rodwell, Phys. Rev. Lett. 75, 4102 (1995).

${ }^{11}$ K. W. Madison, M. C. Fischer, R. B. Diener, Qian Niu, and M. G. Raizen, Phys. Rev. Lett. 81, 5093 (1998).

${ }^{12}$ A. Eckardt, M. Holthaus, H. Lignier, Al Zenesini, D. Ciampini, O. Morsch, and E. Arimondo, Phys. Rev. A 79, 013611 (2009).
} 
${ }^{13}$ S. Longhi, M. Marangoni, M. Lobino, R. Ramponi, P. Laporta, E. Cianci, and V. Foglietti, Phys. Rev. Lett. 96, 243901 (2006).

${ }^{14}$ G. DellaValle, M. Ornigotti, E. Cianci, V. Foglietti, P. Laporta, and S. Longhi, Phys. Rev. Lett. 98, 263601 (2007).

${ }^{15}$ A. Szameit, I. L. Garanovich, M. Heinrich, A. A. Sukhorukov, F. Dreisow, T. Pertsch, S. Nolte, A. Tünnermann, S. Longhi, and Y. S. Kivshar, Phys. Rev. Lett. 104, 223903 (2010).

${ }^{16}$ K.-H. Ahn, H. C. Park, and B. Wu, Physica E 34, 468 (2006).

${ }^{17}$ E. Papp, C. Micu, and L. Aur, Superlattices Microstruct. 44, 770 (2008).

${ }^{18}$ M. Sillanpää, T. Lehtinen, A. Paila, Y. Makhlin, and P. Hakonen, Phys. Rev. Lett. 96, 187002 (2006).

${ }^{19}$ N. Tsuji, T. Oka, and H. Aoki, Phys. Rev. B 78, 235124 (2008).

${ }^{20}$ A. Mysyrowicz, D. Hulin, A. Antonetti, A. Migus, W. T. Masselink, and H. Morkoç, Phys. Rev. Lett. 56, 2748 (1986).

${ }^{21}$ S. G. Carter, V. Birkedal, C. S. Wang, L. A. Coldren, A. V. Maslov, D. S. Citrin, and M. S. Sherwin, Science 310, 651 (2005).

${ }^{22}$ R. Huber, B. A. Schmid, Y. R. Shen, D. S. Chemla, and R. A. Kaindl, Phys. Rev. Lett. 96, 017402 (2006).

${ }^{23}$ J. R. Danielson, Y.-S. Lee, J. P. Prineas, J. T. Steiner, M. Kira, and S. W. Koch, Phys. Rev. Lett. 99, 237401 (2007).

${ }^{24}$ S. Leinß. T. Kampfrath, K. V. Volkmann, M. Wolf, J. T. Steiner, M. Kira, S. W. Koch, A. Leitenstorfer, and R. Huber, Phys. Rev. Lett. 101, 246401 (2008).

${ }^{25}$ M. Kroner, A. O. Govorov, S. Remi, B. Biedermann, S. Seidl, A. Badolato, P. M. Petroff, W. Zhang, R. Barbour, B. D. Gerardot, R. J. Warburton, and K. Karrai, Nature (London) 451, 311 (2008).

${ }^{26}$ H. Hirori, M. Nagai, and K. Tanaka, Phys. Rev. B 81, 081305(R) (2010).

${ }^{27}$ K. Shinokita, H. Hirori, M. Nagai, N. Satoh, Y. Kadoya, and K. Tanaka, Appl. Phys. Lett. 97, 211902 (2010).

${ }^{28}$ M. Wagner, H. Schneider, D. Stehr, S. Winnerl, A. M. Andrews, S. Schartner, G. Strasser, and M. Helm, Phys. Rev. Lett. 105, 167401 (2010).

${ }^{29}$ B. Bartal, I. Z. Kozma, A. G. Stepanov, G. Almási, J. Kuhl, E. Riedle, and J. Hebling, Appl. Phys. B 86, 419 (2007).

${ }^{30}$ N. Karpowicz, J. Dai, X. Lu, Y. Chen, M. Yamaguchi, H. Zhao, X.-C. Zhang, L. Zhang, C. Zhang, M. Price-Gallagher, C. Fletcher, O. Mamer, A. Lesimple, and K. Johnson, Appl. Phys. Lett. 92, 011131 (2008).

${ }^{31}$ H. Hirori, A. Doi, F. Blanchard, and K. Tanaka, Appl. Phys. Lett. 98, 091106 (2011).

${ }^{32}$ M. M. Dignam, Phys. Rev. B 59, 5770 (1999).

${ }^{33}$ T. Meier, H. J. Kolbe, A. Thränhardt, G. Weiser, P. Thomas, and S. W. Koch, Physica E 7, 267 (2000).
${ }^{34}$ D. Wang, A. Zhang, L. Yang, and M. M. Dignam, Phys. Rev. B 77, 115307 (2008).

${ }^{35}$ K. Yashima, K. Oka, K. Hino, N. Maeshima, and X. M. Tong, Solid State Commun. 149, 229 (2009).

${ }^{36} \mathrm{ac}$ Zener tunneling refers to the band-to-band tunneling caused by a strong ac field. In the concerned system, two photon sidebands belonging to different joint SL minibands are coupled to each other by ac Zener tunneling. As regards an exciton state, each photon sideband forms an exciton manifold composed of discrete levels and continua. FR occurs, when the discrete level supported by one photon sideband is degenerate with the continua associated with the other photon sidebands, followed by ac Zener tunneling between the two photon sidebands. To be precise, the ac Zener tunneling couples these two photon sidebands relevant to the exciton states rather than the exciton states themselves in a direct manner. In order to avoid such confusion regarding terminology, hereafter the interaction between the exciton states is termed ac Zener coupling, not ac Zener tunneling.

${ }^{37}$ S. Glutsch and F. Bechstedt, Phys. Rev. B 60, 16584 (1999).

${ }^{38}$ A. Kukuu, T. Amano, T. Karasawa, N. Maeshima, and K. I. Hino, Phys. Rev. B 64, 075318 (2010).

${ }^{39}$ R.-B. Liu and B.-F. Zhu, J. Phys.: Condens. Matter 12, L741 (2000).

${ }^{40}$ K. I. Hino, K. Goto, and N. Toshima, Phys. Rev. B 69, 035322 (2004).

${ }^{41}$ M. V. Fedorov and A. E. Kazakov, Prog. Quantum Electron. 13, 1 (1989).

${ }^{42}$ Z. Deng and J. H. Eberly, Phys. Rev. A 36, 2750 (1987).

${ }^{43}$ P. L. Knight, M. A. Lauder, and B. J. Dalton, Phys. Rep. 190, 1 (1990).

${ }^{44}$ K. I. Hino, X. M. Tong, and N. Toshima, Phys. Rev. B 77, 045322 (2008).

${ }^{45}$ N. Maeshima and K. Hino, Comput. Phys. Commun. 183, 8 (2012).

${ }^{46}$ K. I. Hino, Phys. Rev. B 62, R10626 (2000).

${ }^{47}$ K. I. Hino, Phys. Rev. B 64, 075318 (2001).

${ }^{48}$ V. M. Burke and C. J. Noble, Comput. Phys. Commun. 84, 19 (1994).

${ }^{49}$ A. G. Sunderland, J. W. Heggarty, C. J. Noble, and N. S. Scott, Comput. Phys. Commun. 114, 183 (1998).

${ }^{50}$ A. G. Sunderland, C. J. Noble, V. M. Burke, and P. G. Burke, Comput. Phys. Commun. 145, 311 (2002).

${ }^{51}$ K. Yashima, K. I. Hino, and N. Toshima, Phys. Rev. B 68, 235325 (2003).

${ }^{52}$ U. Fano, Phys. Rev. 124, 1866 (1961).

${ }^{53} \mathrm{H}$. Haug and S. W. Koch, Quantum Theory of the Optical and Electronic Properties of Semiconductors 5th ed. (World Scientific, Singapore, 2009), Chap. 12. 